\title{
Optical topography measurement of steeply-sloped surfaces beyond the specular numerical aperture limit
}

\author{
Matthew Thomas ${ }^{\mathrm{a}}$, Rong Su*a, Peter de Groot ${ }^{\mathrm{b}}$, Richard Leach ${ }^{\mathrm{a}}$ \\ ${ }^{a}$ University of Nottingham, Nottingham, NG8 1BB, UK \\ bZygo Corporation, Laurel Brook Road, Middlefield, CT 06455
}

\begin{abstract}
Engineered functional surfaces often feature varying slopes on macro- and micro-scales. When surfaces are mirror-like, the highest surface slope that can be measured by a far-field 3D imaging optical surface measuring instrument is the arcsine of the numerical aperture (NA) of the objective lens, i.e. the acceptance angle of the lens. However, progress in instrument design has allowed for measurement of non-specular surfaces with slopes steeper than this "traditional" NA limit. Nonetheless, there is currently a lack of understanding about the instrument response to surfaces with steep slopes beyond this limit. It is unclear over what surface spatial frequencies we can expect to accurately report fine surface-feature details. Here we present results demonstrating the capability of a commercial coherence scanning interferometer for measuring surface topography of a roughened flat and a blazed grating with tilt angles greater than the NA slope limit. We show that the surface form, i.e. the tilted plane, can be measured correctly. But, while surface texture information that can appear useful is also obtained, tilting significantly influences the measurement accuracy of micro-scale texture, and for asymmetric gratings, can depend on the tilting direction. A simplified surface scattering model suggests that the loss of scattered power captured by the instrument and a low signal-to-noise ratio causes the reduction of measurement accuracy. However, a rigorous three-dimensional instrument model is needed for a full understanding; we will develop this in our future work.
\end{abstract}

Keywords: optical metrology, surface topography, coherence scanning interferometry, numerical aperture limit, optical modelling

\section{INTRODUCTION}

The topographies of functional surfaces on manufactured parts can have significant effects on the parts' functional properties, performance and lifetime ${ }^{1}$. For example, the surface topography significantly influences the tribological properties and lifetime of a three-dimensional (3D) printed knee replacement, the fuel flow cavitation of a fuel injector nozzle, and the aerodynamic properties and cooling effects of a turbine blade with micro-scale cooling holes ${ }^{1,2}$. To provide comprehensive information about a surface's topography, or to conduct quality inspection of surface-engineered parts, surface topography measurement methods are required ${ }^{3}$. Compared to traditional mechanical contact methods, optical techniques have many advantages, such as their non-contact nature, and the ability of areal optical methods to rapidly capture entire fields of view ${ }^{4}$.

Engineered functional surfaces often feature varying slopes on macro- and micro-scales. For a mirror-like surface that only reflects light in the specular direction, the highest surface slope that can be measured by a far-field 3D optical surface measuring instrument is determined by the numerical aperture (NA) of the objective lens. Light that is specularly reflected by a surface that has a slope larger than one-half of the maximum reflected ray angle $\left(\theta=\sin ^{-1} A_{n}\right.$, where $A_{n}$ is the NA) will fall outside of the acceptance cone of the instrument and not be captured ${ }^{4}$, as shown in Figure 1 . However, many surfaces are not mirror-like and produce a distribution of scatter when illuminated; under the Abbe theory of image formation, it is the capture of this scatter that provides image contrast ${ }^{5,6}$. More information about the object being measured can be recorded in the image if more scattering/diffraction orders can be captured. The limited NA captures only a portion of the scatter, which determines the upper limit to both the resolution of the instrument and to the surface spatial frequencies transferred by the instrument.

* Corresponding author: rong.su@nottingham.ac.uk 

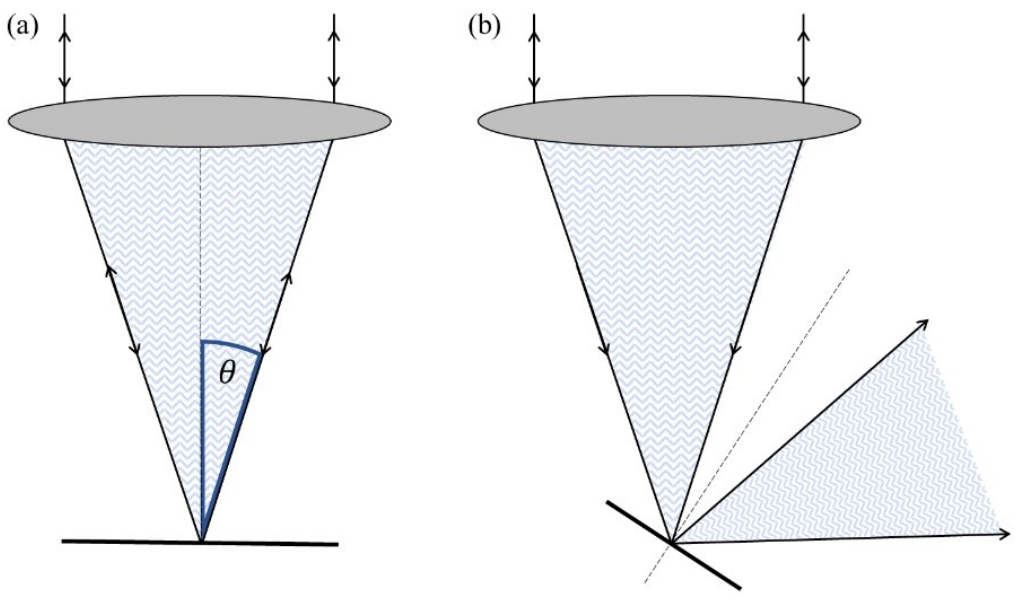

Figure 1. Diagram of the measurement of a mirror-like surface which only specularly reflects under two different tilting arrangements.

Information from steep surfaces beyond the specular limit using surface topography measuring instruments can be acquired by adjusting or making use of multiple viewing angles with respect to the sample, through the use of tilt or multi-axes stages $^{7-10}$, rotating both the sample and the sensor ${ }^{11}$, or making use of multiple sensors ${ }^{12,13}$. Unfortunately, such approaches are comparatively expensive, due to the additional moving parts (or additional sensors), can be slower (moving stage and making multiple measurements), require an accurate combination of measurement data, and have an increased difficulty in retaining stability during a measurement.

However, various instruments are capable of imaging and obtaining topography information from surfaces with slope angles well outside the specular acceptance cone, without repositioning the sample or instrument. This capability is attributed to the capture and detection of diffuse and back-scattered light from the microstructures found on the surface slopes $^{14-17}$, which is possible due to recent advances in instrument technology and design. For a coherence scanning interferometry (CSI) instrument, high dynamic range measurement of parts with wide reflectance ranges can be performed through the alternation of light levels during a measurement or between sequential measurements, and dynamic noise reduction for detection of weak signals can be achieved through signal oversampling ${ }^{17-19}$. In a focus variation instrument, the use of ring light illumination can essentially increase the illumination NA, consequently extending the range of measurable steep surfaces ${ }^{15,20}$.

Typically, image formation from surface slopes that are steep enough that specular reflections are lost, obtain surface information with a low signal-to-noise ratio (SNR), which can lead to non-measured points ${ }^{4,21}$ and high uncertainty. There is currently a lack of understanding about the instrument response to surfaces with steep slopes outside the NA cone, and in particular it is unclear over what surface spatial frequencies we can expect to accurately report texture and fine surfacefeature details. The reliability of topography from these regions is unknown. In this paper, we will present the topography results obtained from measurement of steep and tilted surfaces outside the traditional NA acceptance cone, to demonstrate the capability of a CSI instrument to capture surface information. We will look at both the form and texture components of the surface topography produced, displaying the resulting measurement data for the samples measured when tilted. In comparison to reference measurements, such as CSI measurements of the surface while untilted and from an atomic force microscopy (AFM) instrument, we will discuss problems seen with the surface texture obtained from measurements as a surface is tilted. We also propose a methodology to improve the understanding of these phenomena through rigorous optical models of surface scattering and CSI imaging. A preliminary result is shown - more rigorous and comprehensive investigations will be conducted in our future work. 


\section{METHODS AND MATERIALS}

\subsection{CSI instrument}

We use a Zygo Nexview ${ }^{\mathrm{TM}}$ NX2 CSI instrument with objective lenses outlined in Table 1 for the measurements presented in this paper. A detailed description of the working principle of $\mathrm{CSI}^{22}$ and recent studies on its $3 \mathrm{D}$ transfer function can be found elsewhere ${ }^{23,24}$.

Table 1. CSI instrument's objective lenses used in this work

\begin{tabular}{|l|l|l|}
\hline Magnification & $20 \times$ & $50 \times$ \\
\hline NA & 0.40 & 0.55 \\
\hline Acceptance half-angle from arcsin(NA) & $23.6^{\circ}$ & $33.4^{\circ}$ \\
\hline FOV & $(0.43 \times 0.43) \mathrm{mm}$ & $(0.17 \times 0.17) \mathrm{mm}$ \\
\hline Optical resolution (Sparrow criterion) & $0.71 \mu \mathrm{m}$ & $0.52 \mu \mathrm{m}$ \\
\hline Spatial sampling & $0.43 \mu \mathrm{m} / \mathrm{pixel}$ & $0.17 \mu \mathrm{m} / \mathrm{pixel}$ \\
\hline
\end{tabular}

\subsection{Test samples}

For the sake of simplicity, two planar surfaces with microstructures are used to demonstrate the capability of the instrument to obtain information from surfaces titled beyond the acceptance cone. The first surface is a randomly roughened metal flat, with an $S q$, areal root-mean-square roughness parameter, of $237 \mathrm{~nm}$ evaluated over six regions with standard deviation of $17 \mathrm{~nm}$. All surface parameters in this paper for measurements taken by the CSI instrument using the $20 \times 1$ lens are calculated from the surface texture, obtained from the topography from the following procedure: Gaussian S-filter with a nesting index of $2.5 \mu \mathrm{m}$; levelling by rotation of least-squares mean plane; and Gaussian L-filter with a nesting index of $250 \mu \mathrm{m}$. The second surface used is a blazed grating, specifically a Thorlabs GR13-0305 visible ruled reflective diffraction grating, with a groove spatial frequency of 300 lines per millimetre. The grating substrate is made of soda lime glass, coated with an aluminium reflective coating. Using an AFM instrument in tapping mode, the grating was found to have a pitch of approximately $3.4 \mu \mathrm{m}$, and a peak-to-valley (PV) height of approximately $0.2 \mu \mathrm{m}$, as shown in Figure 2; these parameters are used for modelling scatter from the grating. The AFM instrument used a tip with a maximum radius of $12 \mathrm{~nm}$, obtaining topography over a $(10 \times 10) \mu \mathrm{m}$ region with sample spacing of $26.1 \mathrm{~nm}$, and with a $\mathrm{Z}$ sensor noise level of around $30 \mathrm{pm}$ root-mean-square.

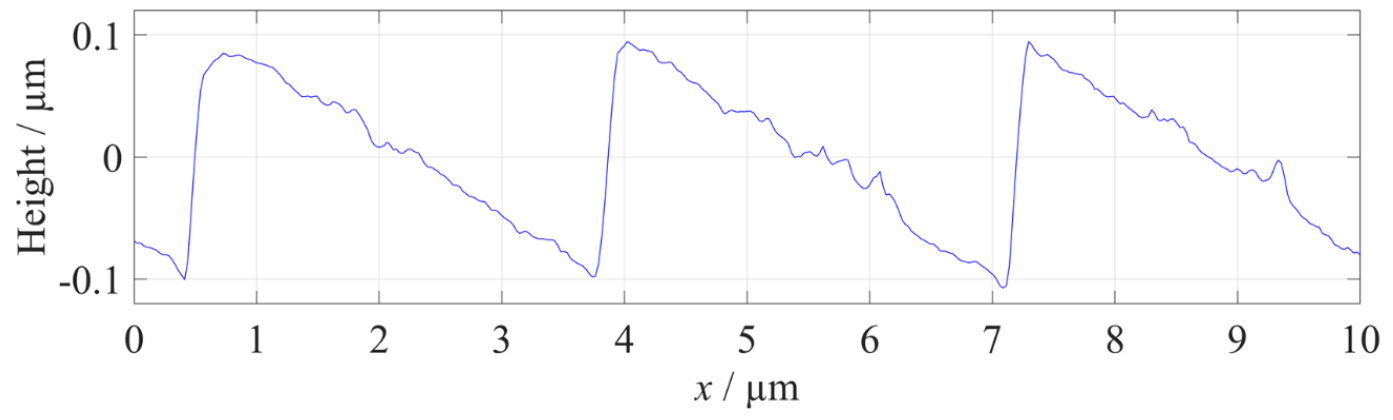

Figure 2. The profile of the GR13-0305 blazed grating as measured by an AFM instrument.

\subsection{Experimental design}

The roughened flat is measured with the $20 \times$ lens while tilted by $30^{\circ}$, to examine the effect on the surface topography information obtained when the collection of the specular scatter is prevented. We expect to still obtain useful topography information from both the surface form (determined by the tilt) and the surface texture. The blazed grating is measured with the $50 \times$ lens at both $0^{\circ}$ and $45^{\circ}$ tilt, as shown in Figure 3; note that case III is symmetrically equivalent to a $45^{\circ}$ tilt in the opposite direction to case II. As a blazed grating produces well defined and separated diffraction orders, and is known for its asymmetric distribution of scatter, a comparison of tilts with the same magnitude but different directions allows us to investigate the connection between the surface texture obtained and the diffraction orders captured while controlling for 
the absolute tilt. While not shown here, the surface homogeneity of the grating was confirmed to allow comparison of measurements from different regions as though they were taken from the same region, and repeat measurements at specific tilts were taken to confirm that the topography results obtained at that tilt were independent of the measurement location.

(a) Case I

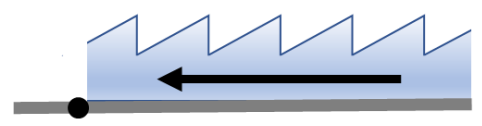

(b) Case II

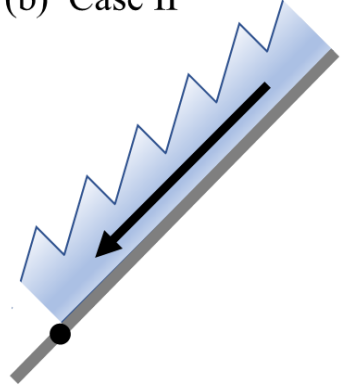

(c) Case III

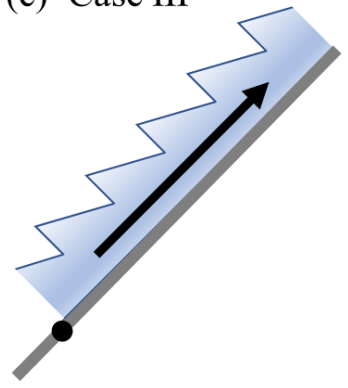

Figure 3. Diagram describing the three tilting arrangements used for measurement, where the black arrow denotes the blaze direction of the grating. (a) Case I: The sample is not titled, (b) Case II: the sample is tilted at $45^{\circ}$ with downward facing blaze, (c) Case III: the sample is tilted at $45^{\circ}$ with upward facing blaze.

\subsection{Diffraction by tilted blazed grating}

Blazed gratings produce an asymmetric distribution of scatter. Consequently, under the Abbe theory of imaging we would expect measurements of the gratings taken at the same absolute tilt but in each direction to produce different results. In order to quantify a blazed grating's scatter distribution, a boundary element method (BEM) scattering model was used. Our BEM model has previously been reported: evidence of its validity and its use in modelling CSI is given elsewhere ${ }^{25}$. For monochromatic, linearly polarised plane wave illumination, the model solves linear partial differential equations along the surface boundary to obtain the field and its surface normal derivative, from which far-field scatter can then be calculated ${ }^{26}$.

In this work, we used a two-dimensional (2D) BEM model to simulate the scattering by a blazed grating at different tilt angles when illuminated by a monochromatic plane wave at normal incidence. Intensity values are obtained from both sand p-polarised illumination, and averaged together, to represent the result of unpolarised illumination. The model is limited to modelling of surfaces of homogenous refractive index with surfaces fully described by lines on the plane of incidence ( $x$-z plane). The intention was to use the basic scattering phenomenon to partially understand the effects that we observed in the CSI surface measurement.

\section{RESULTS}

Despite the $23.6^{\circ}$ acceptance angle of the $20 \times$ objective lens used, in Figure 4(a) and (d) the instrument is shown capable of obtaining topography from the measurement of the $30^{\circ}$ tilted roughened flat, providing form data with surface inclination agreeing closely with the chosen tilt. Similar results on instrument capability can be seen in references ${ }^{17,18}$. Clearly, even the loss of the specular component of the scatter does not prevent useful topography information from being obtained, and that this information must originate from the non-specular scatter captured.

Presented in Figure 4(b) is the texture information obtained from the surface while tilted. Measurements of this tilted surface from six different locations provide a $S q$ of $246 \mathrm{~nm}$ with a standard deviation of $34 \mathrm{~nm}$, which is similar to that obtained at $0^{\circ}$ tilt (described in section 2.2), and this similarity is shown in Figure 4 between plots (b) and (c). However, comparison of the surface topography from the same location while at different tilts is difficult as it is a random surface, and we, therefore, cannot conclude from this result alone whether or not the texture has been accurately measured. To investigate how the loss of scatter affects the measured surface texture, we need to measure a grating which contains periodic and well-defined surface structure. 
(a)
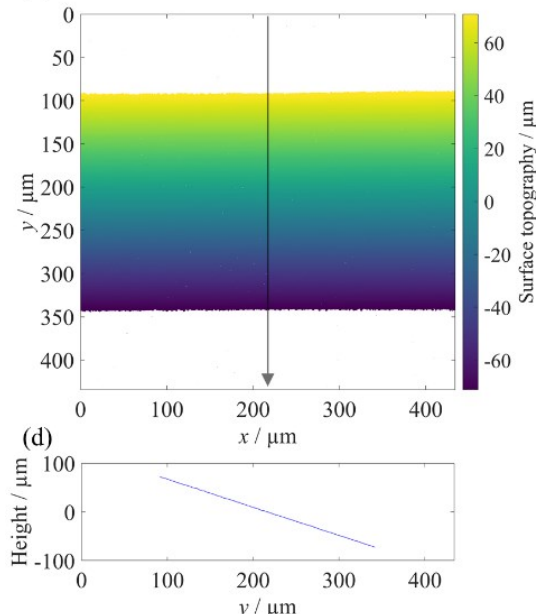

(b)
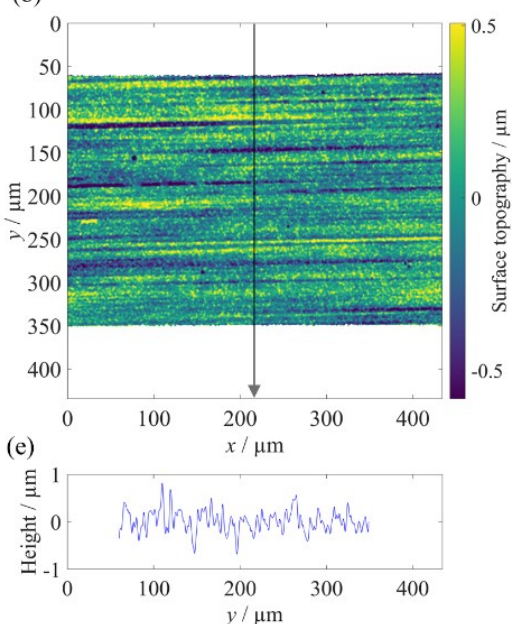

(c)
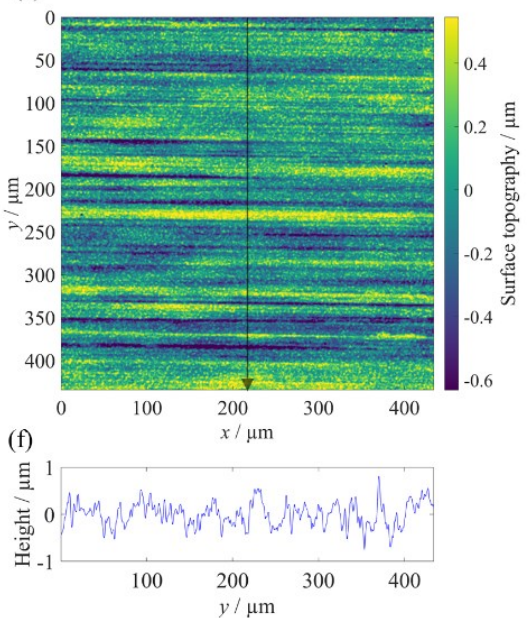

Figure 4. Topography results from a $20 \times$ lens of the roughened flat (a,b,d,e) when tilted at $30^{\circ}$, and (c, f) when untilted. Shown is the (a) raw topography obtained when the surface was tilted, (b) the topography after levelling with the least-squares best fit plane, and (c) the raw topography obtained when the surface was untilted. Plots (d-f) are the north-south profiles taken from plots (a-c) respectively, as marked by the black arrows.

Shown in Figure 5 are the topography results from the measurement of the blazed grating using the $50 \times$ lens corresponding to cases I, II and III as defined in Figure 3.
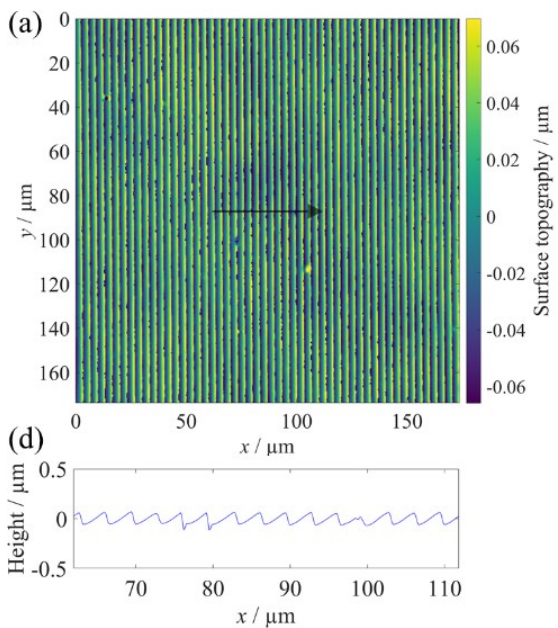
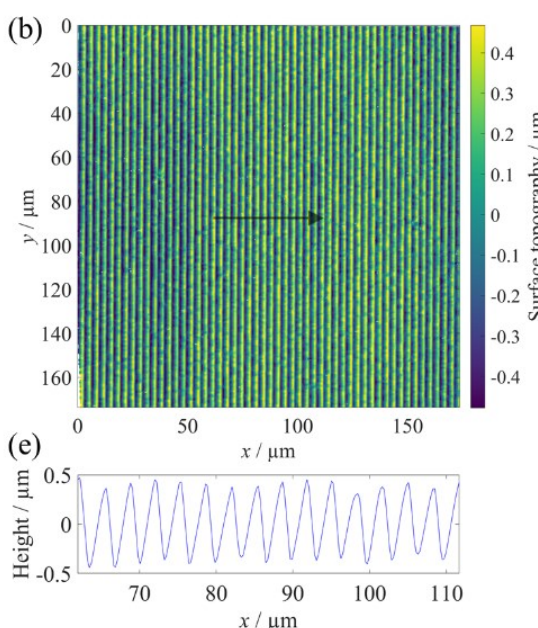
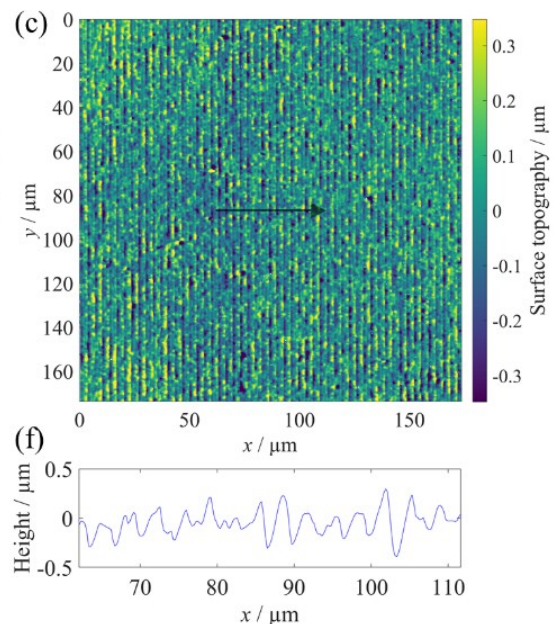

Figure 5. Topography results after plane form removal from a CSI measurement of a GR13-0305 blazed grating with tilting arrangements of (a) Case I, (b) Case II, and (c) Case III, using a 50× lens; corresponding profiles are shown below in plots (d-f). These profiles are taken along and in the direction of the lines marked by the black arrows.

These results show that the texture information obtained from the blazed grating is influenced significantly by tilting, and the tilting direction chosen. From the $0^{\circ}$ results shown in Figure 5(a), the grating's blazed profile is shown, however, the PV heights across the profile are approximately $0.12 \mu \mathrm{m}$, below the $0.2 \mu \mathrm{m}$ PV height measured by the AFM instrument. This reduction in height is expected and occurs due to the spatial frequency transfer characteristics of the CSI instrument ${ }^{27}$ 29,23 , with a transmission magnitude at $0.3 \mu \mathrm{m}^{-1}$ of approximately $70 \%{ }^{24}$. Further reductions to the transmittance are expected as the instrument will have had some reference mirror defocus present while the measurements were taken ${ }^{30}$.

Despite being measurements of the same surface and at the same absolute tilt, the results from case II and case III differ significantly; the topography from case II retains the overall blazed structure, while case III provides significantly less visible structure. This difference occurs due to the asymmetry of scatter from the grating. The simulated far-field 
scattering/diffraction of an ideal blazed grating with geometry matching that of the GR13-0305 under normally incident monochromatic plane wave illumination of wavelength $580 \mathrm{~nm}$ (the central wavelength of the CSI system) is shown in Figure 6. Clearly, more scattered power is collected within the aperture and a high SNR is achieved for case II (Figure 6(b)) compared to case III (Figure 6(c)).

Although the measurement results in case II retain a blazed grating structure with the correct pitch, the PV height of the blazed structure is approximately $0.82 \mu \mathrm{m}$, around four times larger than the value from the AFM measurement. Clearly, the CSI measurement of case I $\left(0^{\circ}\right.$ tilt) provides a result that is much closer to the reference measurement. This result is consistent with the scattering modelling in Figure 6(a) where more diffraction orders are captured at a high SNR within the NA limit.

However, from these results we cannot entirely understand why the measured grating's PV height and surface height variation are amplified in cases II and III. A fully 3D rigorous CSI model that considers polychromatic incoherent illumination, based on the recently reported boundary source method surface scattering model $^{31}$, is required (an extension of the $2 \mathrm{D}$ rigorous $\mathrm{CSI}$ mode $^{25}$ ).
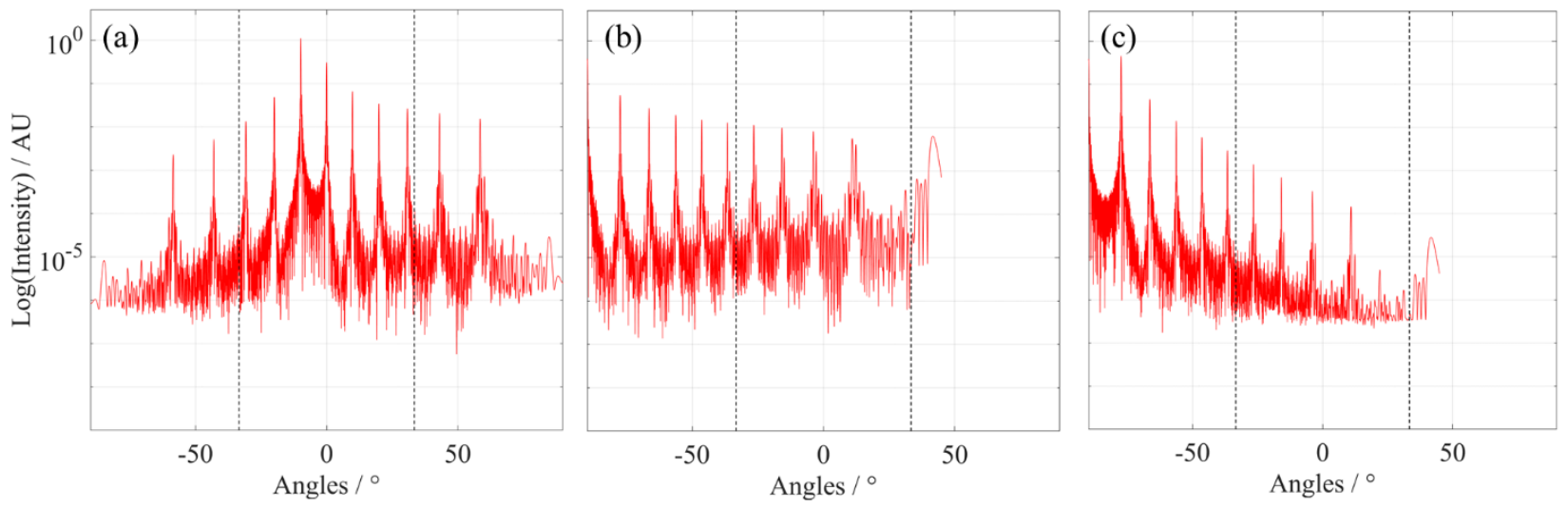

Figure 6. Modelling results for scattered intensity from the blazed grating shown in Figure 5, for the average intensity obtained from both s- and p-polarised monochromatic illumination of wavelength $580 \mathrm{~nm}$, (a) Case I, (b) Case II, and (c) Case III. The region between the dashed black lines denote the $\pm 33.4^{\circ}$ acceptance cone for the $50 \times$ lens $(0.55 \mathrm{NA})$.

\section{CONCLUSION}

In this paper, while instruments traditionally struggle with measuring outside their NA limited acceptance cone, we have shown that the state-of-the-art CSI instruments can now be sensitive enough to obtain topographic information for tilted surfaces well beyond the traditional acceptance angle. While surface texture information that can appear useful is also obtained, we have shown that tilting significantly influences the measurement accuracy of micro-scale texture, and for asymmetric gratings can depend on the tilting direction. From our modelling results, we suggest that the loss of scattered power captured by the instrument and a low SNR would cause reduction of measurement accuracy; however, a rigorous 3D CSI model is needed for a full understanding and this is the focus of our future work.

\section{FUNDING \& ACKNOWLEDGEMENTS}

This work was supported by the Engineering and Physical Sciences Research Council [grant number EP/M008983/1] (EPSRC); and the European Union's Horizon 2020 Research and Innovation Programme (MNR4SCell, 734174). The authors would like to thank Dr Mingyu Liu from the University of Nottingham for the AFM measurement of the blazed grating, to Prof. Jeremy Coupland and Dr Nikolay Nikolaev from Loughborough University for the use of the BEM code, and to Dr Xavier Colonna de Lega from Zygo for his valuable discussion and feedback. 


\section{REFERENCES}

[1] Bruzzone, A. A. G., Costa, H. L., Lonardo, P. M. and Lucca, D. A., "Advances in engineered surfaces for functional performance," CIRP Ann. 57(2), 750-769 (2008).

[2] Echouchene, F., Belmabrouk, H., Le Penven, L. and Buffat, M., "Numerical simulation of wall roughness effects in cavitating flow," Int. J. Heat Fluid Flow 32(5), 1068-1075 (2011).

[3] Leach, R. K., ed., [Fundamental Principles of Engineering Nanometrology, 2nd ed.], William Andrew Publishing (Elsevier) (2014).

[4] Leach, R. K., ed., [Optical Measurement of Surface Topography], Springer Berlin Heidelberg, Berlin, Heidelberg (2011).

[5] Abbe, E., "VII.- On the estimation of aperture in the microscope.," J. R. Microsc. Soc. 1(3), 388-423 (1881)

[6] Masters, B. R., "Abbe theory of image formation and diffraction of light in transmitted light microscopes," [Confocal Microscopy and Multiphoton Excitation Microscopy: The Genesis of Live Cell Imaging], SPIE Press, Bellingham, Washington, USA, 37-49 (2006).

[7] Marinello, F., Bariani, P., Carmignato, S., Savio, E., De Chiffre, L. and Bossard, M., "Increase of maximum detectable slope with optical profilers: theory and applicative examples," Proc Int Euspen Conf, Bremen, DE (2007).

[8] Jiang, X., Gao, F. and Mateboer, A., "An approach of assessment for ultra-precision v-groove structured surfaces," Proc. 10th Int. Conf. Eur. Soc. Precis. Eng. Nanotechnol. EUSPEN 2010, 512-516, euspen (2010).

[9] Colonna de Lega, X., Dresel, T., Liesener, J., Fay, M. F., Gilfoy, N., Delldonna, K. and de Groot, P., "Optical form and relational metrology of aspheric micro optics," Proc 32nd Annu. Meet. ASPE 67, 20-23, Charlotte, North Carolina, US (2017).

[10] Ren, F., Wang, Z., Qian, J., Liang, Y., Dang, S., Cai, Y., Bianco, P. R., Yao, B. and Lei, M., "Multi-view object topography measurement with optical sectioning structured illumination microscopy," Appl. Opt. 58(23), 6288 (2019).

[11] Conroy, M., Burton, R., Yu, Y. and Kumagi, T., "Recent advances in non-contact metrology, high speed measurement, steep slope measurement and correlation with stylus data," Proc. Malays. Int. Tribol. Conf. 2015, 314-315, Penang, Malaysia (2015).

[12] Zhang, T., Gao, F. and Jiang, X., "Surface topography acquisition method for double-sided near-right-angle structured surfaces based on dual-probe wavelength scanning interferometry," Opt. Express 25(20), 2414824156 (2017).

[13] Zhang, T., Gao, F., Martin, H. and Jiang, X., "A method for inspecting near-right-angle V-groove surfaces based on dual-probe wavelength scanning interferometry," Int. J. Adv. Manuf. Technol. 104(1), 1-7 (2019).

[14] Leach, R. K., de Groot, P. and Haitjema, H., "Infidelity and the calibration of surface topography measuring instruments," Proc 33rd Annu. Meet. ASPE, Las Vegas, USA (2018).

[15] Danzl, R. and Helmli, F., "Three-dimensional reconstruction of surfaces with steep slopes using an optical measurement system based on a colour focus sensor," Proc. 6th Euspen Int. Conf., Baden bei Wien, AT (2006).

[16] Zangl, K., Danzl, R., Helmli, F. and Prantl, M., "Highly accurate optical $\mu$ CMM for measurement of micro holes," Procedia CIRP 75, 397-402 (2018).

[17] Fay, M. F., Colonna de Lega, X. and de Groot, P., "Measuring high-slope and super-smooth optics with highdynamic-range coherence scanning interferometry," Class. Opt. 20142014 Pap. OW1B3, OW1B.3, Optical Society of America (2014).

[18] Fay, M. F., Colonna de Lega, X. and Schmidt, M., "Measuring high-slope parts using coherence scanning interferometry," Proc 29th Annu. Meet. ASPE (2014).

[19] Gomez, C., Su, R., Thompson, A., DiSciacca, J., Lawes, S. and Leach, R. K., "Optimization of surface measurement for metal additive manufacturing using coherence scanning interferometry,” Opt. Eng. 56(11), 111714 (2017).

[20] Hiersemenzel, F., Petzing, J. N., Leach, R. K., Helmli, F. and Singh, J., “Areal texture and angle measurements of tilted surfaces using focus variation methods," 3rd Int. Conf. Surf. Metrol., 5, Annecy, France (2012).

[21] Petzing, J., Coupland, J. M. and Leach, R. K., [The measurement of rough surface topography using coherence scanning interferometry], National Physical Laboratory (2010).

[22] de Groot, P., "Coherence scanning interferometry," [Optical Measurement of Surface Topography], R. K. Leach, Ed., Springer Berlin Heidelberg, Berlin, Heidelberg, 187-208 (2011). 
[23] Su, R., Wang, Y., Coupland, J. M. and Leach, R. K., "On tilt and curvature dependent errors and the calibration of coherence scanning interferometry," Opt. Express 25(4), 3297-3310 (2017).

[24] Su, R., Thomas, M., Liu, M., Drs, J., Bellouard, Y., Pruss, C., Coupland, J. M. and Leach, R. K., "Lens aberration compensation in interference microscopy," Opt. Lasers Eng. 128, 106015 (2020).

[25] Thomas, M., Su, R., Nikolaev, N., Coupland, J. M. and Leach, R. K., "Modelling of coherence scanning interferometry for complex surfaces based on a boundary element method," Model. Asp. Opt. Metrol. VII 11057, 1105713, International Society for Optics and Photonics (SPIE), ICM, Munich, Germany (2019).

[26] Simonsen, I., "Optics of surface disordered systems. A random walk through rough surface scattering phenomena,” Eur. Phys. J. Spec. Top. 181(1), 1-103 (2010).

[27] de Groot, P. and Colonna de Lega, X., "Interpreting interferometric height measurements using the instrument transfer function," Fringe 2005, W. Osten, Ed., 30-37, Springer, Berlin, Heidelberg (2006).

[28] Church, E. L., Dainty, C., Gale, D. M. and Takacs, P. Z., "Comparison of optical and mechanical measurements of surface finish,” Adv. Opt. Manuf. Test. II 1531, 234-250, International Society for Optics and Photonics (SPIE) (1992).

[29] Coupland, J. M. and Lobera, J., "Holography, tomography and 3D microscopy as linear filtering operations," Meas. Sci. Technol. 19(7), 074012 (2008).

[30] Su, R., Thomas, M., Leach, R. K. and Coupland, J. M., "Effects of defocus on the transfer function of coherence scanning interferometry," Opt. Lett. 43(1), 82-85 (2018).

[31] Coupland, J. M. and Nikolaev, N. I., "A new approach to vector scattering: the 3s boundary source method," Opt. Express 27(21), 30380-30395 (2019). 